\title{
Nuevas herramientas para la clasificación taxonómica de los insectos vectores de leishmaniosis: utilidad de los genes mitocondriales
}

\author{
Eduar E. Bejarano \\ Programa de Estudio y Control de Enfermedades Tropicales, PECET, \\ Universidad de Antioquia, Medellín, Colombia.
}

\begin{abstract}
La controversia en torno a la posición taxonómica de los flebotomíneos, debida en parte a dificultades morfológicas, ha evidenciado la necesidad de buscar nuevas herramientas que permitan no sólo establecer una clasificación más acorde con los procesos evolutivos que han experimentado estos insectos, sino también diferenciar los taxones que conforman los complejos de especies. Con la aparición de las técnicas de biología molecular, el estudio del genoma en insectos vectores de enfermedades se ha convertido en un paso importante en la resolución de muchos conflictos taxonómicos. Las características del ADN mitocondrial, entre las que se destacan su rápida evolución y la facilidad con la que se puede aislar y manipular, han favorecido su elección como marcador molecular para estudios de taxonomía. Recientemente, el polimorfismo de algunos genes mitocondriales comenzó a utilizarse para explorar las relaciones filogenéticas y evolutivas en especies de los géneros Lutzomyia y Phlebotomus. La presente revisión recopila la literatura disponible sobre los principales grupos de flebotomíneos estudiados, los genes mitocondriales más utilizados y su contribución a la clasificación de los vectores de leishmaniosis.
\end{abstract}

Palabras clave: ADN mitocondrial, flebotomíneos, taxonomía, filogenética, leishmaniosis.

New tools for the taxonomic classification of the insect vectors of leishmaniasis: usefulness of mitochondrial genes

The controversial taxonomic position of phlebotomine sandflies based upon morphological evidence indicates the need to examine alternative sources of phylogenetic information. New taxonomic tools could lead to a more consistent classification within phlebotomine sandflies according to evolution patterns and, to distinguish morphologically similar species. With the molecular biology techniques now available, the study of insect vector genomes has become an important step to help to resolve many taxonomic problems. The majority of the genes chosen for such studies are mitochondrial because they are easier to isolate and manipulate, and evolve quickly. Recently, mitochondrial gene polymorphisms have begun to be used to infer phylogenetic relationships among species of genera Lutzomyia and Phlebotomus. This article presents an updated review about the main studied phlebotomine groups, selected mitochondrial genes, and its contribution to the classification of leishmaniasis vectors.

Key words: mitochondrial DNA, phlebotomine sandflies, taxonomy, phylogenetic, leishmaniasis.

Dentro de los insectos de importancia médica, la posición taxonómica de los dípteros hematófagos de la familia Psychodidae, subfamilia

Correspondencia:

PECET, Universidad de Antioquia, Cra. 50 A No. 63-85, Medellín, Colombia. Teléfono: 26355 55. Fax: 5716675. bejarano@medicina.udea.edu.co

Recibido: 12/02/01; aceptado: 29/05/01
Phlebotominae, involucrados en la transmisión de leishmaniosis, bartonelosis y arbovirosis, ha sido una de las más controversiales. Mientras algunos autores, como Williams (1), sugieren que los flebotomíneos constituyen un experimento evolutivo diferente a los demás miembros de Psychodidae, lo que amerita darles la posición de familia, otros investigadores como Lewis et al. 
(2) se han mostrado a favor de seguir manteniendo el rango de subfamilia para estos insectos.

Esta controversia obedece en parte a la homogeneidad de los caracteres morfológicos utilizados para establecer los esquemas de clasificación a niveles supraespecíficos, dado que estos insectos han evolucionado con muy pocos cambios en su morfología (3). Por ejemplo, la separación de los más importantes géneros de la subfamilia Phlebotominae del Nuevo y Viejo Mundo, se basa en escasos caracteres morfológicos presentes en el género Lutzomyia França, 1924, y ausentes en Phlebotomus Rondani y Berté, 1840 , entre los que se encuentran el abultamiento en la parte posterior del cibario y el grupo de setas presentes en el episternón $(2,4)$.

Al nivel de especie, los caracteres utilizados se tornan heterogéneos, como lo demuestran las grandes variaciones morfológicas observadas en la terminalia de los machos y espermatecas de las hembras de Lutzomyia que, generalmente, exhiben una morfología especie-específica $(4,5)$. Esta heterogeneidad ha permitido, además de la descripción y el conocimiento de especies, organizar la diversidad flebotomínea en subgéneros, grupos, series y complejos de especies.

A pesar de la gran variación morfológica específica observada en el género Lutzomyia, la determinación de especie en algunos casos sólo puede realizarse en uno de los sexos (5). El grupo verrucarum Theodor, 1965, por ejemplo, se caracteriza porque las hembras de algunas especies son morfológicamente indistinguibles, lo cual ha incidido en el desconocimiento de la posición de algunos componentes del grupo $(5,6)$, en especial, de los taxones agrupados en el complejo townsendi: Lutzomyia townsendi (Ortiz, 1959); Lutzomyia spinicrassa Morales, OsornoMesa, Osorno y Hoyos, 1969; Lutzomyia longiflocosa Osorno-Mesa, Morales, Osorno y Hoyos, 1970; Lutzomyia quasitownsendi Osorno, Osorno-Mesa y Morales, 1972; Lutzomyia sauroida Osorno-Mesa, Morales y Osorno, 1972; Lutzomyia amilcari Arredondo, 1984; Lutzomyia youngi Feliciangeli y Murillo, 1985; y Lutzomyia torvida
Young, Morales y Ferro, 1994. La determinación de especie en este complejo se basa en características morfológicas muy sutiles y sólo puede realizarse en presencia de machos $(5,7)$. Esto tiene importancia epidemiológica al dificultar la identificación de las hembras implicadas en la transmisión de leishmaniosis (8-10). Adicionalmente, los pocos estudios isoenzimáticos del grupo han revelado que $L$. longiflocosa, $L$. sauroida y L. quasitownsendipodrían ser coespecíficas (6).

Las dificultades y controversias expuestas ponen de manifiesto la necesidad de buscar nuevas herramientas para tratar de elucidar los inconvenientes de asignación de los taxa con base en caracteres morfológicos. Estas herramientas deberán ser útiles para discriminar complejos de especies, así como plantear una clasificación más acorde con los procesos evolutivos que han experimentado los flebotomíneos (11). Con ello, la actual clasificación dejaría de ser una abstracción ideal, justificable porque evita la confusión entre estudiantes de este grupo de insectos que no tienen formación en taxonomía $(2,12)$, para convertirse en una clasificación que refleje las relaciones naturales, contribuyendo de paso a una mejor comprensión de la coevolución parásito-vector $(13,14)$.

Con la aparición de las técnicas de biología molecular y, especialmente, de la reacción en cadena de la polimerasa (PCR) y el secuenciamiento automatizado $(15,16)$, el estudio del genoma en insectos vectores de enfermedades se ha convertido en un paso muy importante hacia la resolución de conflictos taxonómicos (1719). En los últimos años, el ADN mitocondrial ha despertado el interés de sistemáticos y entomólogos médicos, por las características particulares de este genoma que lo hacen útil para estudios de genética de poblaciones y filogenética. La presente revisión recopila la literatura disponible sobre los principales grupos de flebotomíneos estudiados, los genes mitocondriales más usados y su contribución a la taxonomía de los vectores de leishmaniosis.

\section{Características del ADN mitocondrial}

El genoma mitocondrial se diferencia del genoma nuclear en características como el número de 
copias, el tamaño, la organización, la herencia y el código genético $(20,21)$. En los insectos, está constituido por una sola molécula circular de ADN de doble cadena, con una longitud aproximada de $16 \mathrm{~kb}$. Posee 13 genes que codifican para proteínas involucradas en el transporte de electrones y fosforilación oxidativa, representados por 3 subunidades del complejo citocromo c oxidasa (COI, COII, COIII), 2 subunidades de la ATPasa (ATPasa 6, ATPasa 8), 7 subunidades del complejo NADH deshidrogenasa (ND1, ND2, ND3, ND4, ND4L, ND5, ND6) y 1 subunidad que hace parte del complejo citocromo c reductasa, denominada citocromo b $(22,23)$.

Adicionalmente, este genoma contiene 22 genes que codifican para ARN de transferencia usados para la traducción de los ARN mensajeros mitocondriales y 2 genes ARN que codifican para la subunidad larga y subunidad pequeña ribosomal (LRU y SPU) relacionadas con el ensamblaje de proteínas (23). Presenta también una región control, no codificante, conocida en los insectos como la región rica en adenina y timina, a través de la cual el núcleo controla la replicación, transcripción y traducción mitocondrial $(24,25)$.

Una de las particularidades más sobresalientes del ADN mitocondrial en insectos, es su alto contenido de adenina y timina, el cual en algunos taxa como Anopheles gambiae Giles, 1902, puede representar hasta el $80 \%$ de toda la molécula (22). Para explicar el alto contenido, se ha postulado la conversión de pares de guanina-citosina hacia adenina-timina mediante alquilación o procesos evolutivos dirigidos por restricciones funcionales y estructurales de la mitocondria que se ha especializado en la producción de aminoácidos hidrofóbicos (fenilalanina, leucina, isoleucina) cuyos codones son ricos en adenina y timina $(26,27)$. Otro aspecto relevante en este genoma es su código genético el cual difiere del código estándar del genoma nuclear (23).

Dentro de las características más importantes que han llevado a la consolidación del ADN mitocondrial como un marcador adecuado para estudios de sistemática molecular $(28,29)$, se destaca la organización simple y uniforme que facilita la comparación entre diversos grupos taxonómicos, la relativa alta tasa de sustitución por carecer de reparasas y la facilidad con la que se puede aislar y manipular $(30,31)$.

Los insectos, como la mayoría de los metazoos, heredan la molécula mitocondrial de forma matrilineal, ya que el espermatozoo de los machos contribuye con muy poco citoplasma al cigoto en formación. Por consiguiente, el genoma mitocondrial del embrión se deriva casi en su totalidad del citoplasma de la madre.

En insectos de importancia médica, la molécula mitocondrial completa ha sido secuenciada en dos especies pertenecientes al género Anopheles: Anopheles gambiae y Anopheles quadrimaculatus (Say, 1824), (22,32). Sin embargo, se han obtenido secuencias nucleotídicas de distintos genes, en especies de los géneros Lutzomyia (33-35), Phlebotomus $(36,37)$, Aedes Meigen, 1818 $(38,39)$, Culex Linnaeus, $1758(40,41)$, Simulium Latreille, 1802 (19,42,43), Triatoma Laporte, 1832 (44), Rhodnius Stal, 1859 (45), Pastrongylus Berg, 1879 (18), entre otros. Para resolver disputas taxonómicas se ha usado la diferencia en la organización de los genes, la divergencia en las secuencias nucleotídicas o aminoacídicas, el polimorfismo en la longitud de los fragmentos de restricción (RFLP) y el polimorfismo conformacional de cadena sencilla (SSCP).

\section{Estudios de genética de poblaciones}

Los haplotipos mitocondriales, definidos como secuencias nucleotídicas o amino acídicas que difieren de una secuencia homóloga por, al menos, una sustitución nucleotídica o el remplazo de un aminoácido, han sido muy útiles para estudiar la variabilidad genética en poblaciones naturales y estimar los niveles de flujo genético entre dichas poblaciones (46). Debido a que el ADN mitocondrial exhibe considerables niveles de variación entre poblaciones naturales, ha mostrado ser un excelente marcador de estructura de poblaciones, revelando patrones de variación geográfica intraespecífica (30).

Los genes utilizados incluyen la región 3' de la subunidad 4 de la NADH deshidrogenasa (ND4), usada para explorar la posición taxonómica de $L$. longipalpis (Lutz y Neiva, 1912), principal vector 
de leishmaniosis visceral en América (35). La divergencia nucleotídica expresada en 149 sitios polimórficos $(24,1 \%)$ del fragmento amplificado de 647 pares de bases $(\mathrm{pb})$, confirmó la existencia de cuatro morfoespecies al interior del taxón, presentes en: 1) Brasil, 2) Colombia (Huila, Cundinamarca) y Venezuela, 3) Colombia (Santander), y 4) Centroamérica (Honduras, Guatemala, Costa Rica). Los resultados señalaron cambios significativos entre las secuencias de los diferentes países (60-63 sustituciones) y bajos niveles de flujo genético entre dichas poblaciones.

Así mismo, la población de L. longipalpis de Girón, Santander, apareció sorprendentemente distinta de las demás poblaciones de Colombia, Venezuela y Brasil, presentando 41 sustituciones nucleotídicas en contraste a las 3-4 sustituciones observadas entre las poblaciones de Cundinamarca y Huila. Con base en el concepto de especie filogenética, la divergencia nucleotídica expresada en ND4, reveló que en Colombia existen, por lo menos, dos especies del complejo L. longipalpis (47).

Un fragmento de $380 \mathrm{pb}$ correspondiente al gen que codifica para la SRU, fue amplificado y secuenciado por Uribe et al. 1998 (26) en individuos de seis poblaciones de L. longipalpis de Colombia (Cundinamarca y Santander), Venezuela (Trujillo), Brasil (Ceará y Minas Gerais), y Guatemala (EI Progreso), incluyendo además una población de Lutzomyia gomezi (Nitzulescu, 1924) de Colombia (Antioquia) como grupo externo. Al comparar las secuencias nucleotídicas de $L$. longipalpis, se encontraron 16 sitios variables $(4,2 \%)$, lo cual sugiere que los genes ribosomales mitocondriales evolucionan lentamente en Lutzomyia, cuando se comparan con la tasa de sustitución que poseen algunos genes codificadores de proteínas como ND4. Esta lenta evolución ribosomal se ha reportado, además, en insectos de otras familias y órdenes $(18,19,45)$.

El complejo L. longipalpis fue estudiado también por Arrivillaga et al. 1999 (48) comparando diez poblaciones de Sur y Centroamérica mediante tres regiones mitocondriales: COI, LRU y SRU. La diversidad genética entre las poblaciones fue evaluada con base en el polimorfismo conformacional de cadena sencilla (SSCP). El análisis de SSCP reveló 19 haplotipos para COI, 14 haplotipos para SRU, y 7 haplotipos para $\mathrm{LRU}$, que soportaron la existencia de cuatro linajes o especies al interior de $L$. longipalpis, presentes en: 1) Centroamérica, 2) Brasil, 3) Los Andes y 4) región central andina. Las diferencias en el número de haplotipos detectados para cada gen, son un reflejo de las distintas tasas de sustitución nucleotídica que estos poseen, y confirman que las subunidades ribosomales evolucionan lentamente en Lutzomyia. Esto sugiere también que dichos genes, por ser relativamente conservados, podrían ser útiles para estudios filogenéticos a niveles taxonómicos profundos.

Actualmente, la secuencia nucleotídica del extremo 3' del gen ND4 está siendo usada para estudiar el flujo de genes al interior de Lutzomyia evansi (Nuñez-Tovar, 1924), vector de leishmaniosis visceral, comparando poblaciones naturales de zonas rurales y urbanas, con presencia y ausencia de la enfermedad (49). El análisis preliminar de las secuencias revela la existencia de flujo genético entre las distintas poblaciones de la especie en la costa Caribe colombiana, y un aumento de la divergencia nucleotídica cuando se comparan poblaciones distanciadas geográficamente (Colombia y Venezuela). Esto fue observado también al estudiar poblaciones de L. ovallesi (Ortiz, 1952) de Colombia y Guatemala (Bejarano y Rojas, 2001, datos no publicados), acentuán-dose más la divergencia cuando el nivel de comparación es interespecífico, lo cual valida la utilidad de ND4 como marcador molecular para estudiar la variabilidad genética en estos dos vectores.

El gen mitocondrial que codifica para la proteína citocromo $b$, se ha usado ampliamente en estudios de genética de poblaciones $(29,31)$ incluyendo varios taxa de Phlebotominae. Montoya et al. (50) utilizaron secuencias de este gen para explorar la estructura de poblaciones de $L$. evansi en Colombia y Venezuela. Éstas confirmaron la posición taxonómica de la especie ubicándola en la serie verrucarum y revelaron, con base en el reloj molecular mitocondrial, que dichas poblaciones divergieron en el Pleistoceno, desde hace 1,5 millones de años. 
Ready et al. (33) compararon haplotipos del gen citocromo b en L. whitmani (Antunes y Coutinho, 1939) de Brasil, y sugirieron la existencia de tres linajes en el taxón. Las especies filogenéticas fueron asociadas a tres zonas bioclimáticas distintas: el linaje del sureste a regiones de bosque amazónico tropical, el linaje del norte-sur a zonas de sabanas del interior de Brasil y el linaje del noroeste a una región de bosque tropical en la Costa Atlántica de este país (51). Calibrando el reloj molecular mitocondrial para flebotomíneos, se estableció que los linajes aparecieron probablemente a finales del Plioceno (2-3 millones de años atrás), época en la cual $L$. whitmani se separó y permaneció en las tres zonas bioclimáticas que sostienen actualmente el complejo de especies. Ishikawa et al. (52) utilizaron secuencias del mismo gen para demostrar el origen monofilético de las poblaciones de $L$. whitmanipresentes en regiones de selva lluviosa amazónica y en la región nororiental de Brasil, encontrando 26 sitios variables en el fragmento amplificado de $294 \mathrm{pb}$, que corresponde al extremo 3' de citocromo b.

Más recientemente, el gen citocromo $b$ ha sido usado para explorar la variación intraespecífica en $L$. youngi, una especie isomórfica perteneciente al complejo townsendi, registrada en Colombia, Venezuela y Costa Rica. Los haplotipos mitocondriales han corroborado la identidad de las poblaciones de L. youngi presentes en el Valle del Cauca, al compararlas con secuencias de la localidad tipo en Las Calderas (Venezuela), confirmando además la presencia de una nueva población de dicha especie en Colombia, en el departamento de Caldas (49).

Dentro del género Phlebotomus, Essingher et al. (36) reportaron el uso de un fragmento del ADN mitocondrial que abarca desde el extremo 3' del gen citocromo $b$ hasta la subunidad 1 de la NADH deshidrogenasa, separados por un gen RNA de transferencia (serina) y dos espaciadores intergénicos, para estudiar la estructura de poblaciones de P. papatasi (Scopoli, 1786), vector de Leishmania major (Yakimoff y Schokhor, 1914). La divergencia nucleotídica en el fragmento amplificado de $441 \mathrm{pb}$, sugirió una reciente radiación de la especie desde la subregión oriental mediterránea, hasta el norte de África, sur de Europa y occidente de Asia. Esta divergencia asociada a datos geográficos y demográficos, permitió no sólo conocer cuales fueron las regiones que ocuparon las poblaciones ancestrales a partir de las cuales se dispersó la especie, sino también, calcular el tiempo de divergencia de dichas poblaciones, el cual fue estimado entre $135.000 \mathrm{y}$ 66.100 años. Este trabajo, considerado como pionero en la exploración de patrones demográficos en Phlebotominae, sirvió para demostrar la utilidad adicional de los genes mitocondriales en estudios de filogeografía con vectores de leishmaniosis.

\section{Estudios de filogenética}

Las secuencias nucleotídicas de porciones selectas del genoma mitocondrial han revolucionado el estudio de las relaciones evolutivas entre especies y altos taxa, actividad que fundamenta y constituye uno de los principales objetivos de la sistemática filogenética (53). Las diferencias en la tasa de sustitución de algunos genes, como las subunidades ribosomales, la región control y los codificadores de proteínas, permiten disponer de una o más regiones mitocondriales, cuya utilidad depende del nivel taxonómico para estudiar $(28,31)$. Estas diferencias han sido muy útiles para establecer relaciones filogenéticas en diversos grupos taxonómicos, al nivel de especie, subgénero, género, familia y orden.

Suguri et al. (54) secuenciaron un segmento de $441 \mathrm{pb}$ del gen que codifica para la enzima COI, en un intento por explorar la variación interespecífica en tres especies del género Lutzomyia: L. ayacuchensis Cáceres y Galati, 1988, L. hartmanni (Fairchild y Hertig, 1957) y L. trapidoi (Fairchild y Hertig, 1952). El alineamiento de las secuencias reveló 65 posiciones nucleotídicas variables, las cuales podrían ser usadas para la identificación molecular de dichas especies. Posteriormente, Sanjoba et al. (37) también utilizan el polimorfismo expresado en el gen COI, para diferenciar cuatro especies de Phlebotomus: P. papatasi, P. major, P. sergenti 
Parrot, 1917, y P. simici Nitzulescu y Nitzulescu, 1931. Las secuencias obtenidas (314 pb) mostraron una similaridad del 80,8 a $85,3 \%$, concluyendo que las divergencias encontradas podrían servir para discriminar las especies en estudio.

Secuencias nucleotídicas del extremo 3' del gen ND4, están siendo usadas para estudiar las relaciones filogenéticas y evolutivas entre algunas de las especies que conforman el grupo verrucarum como L. columbiana (Ristorcelli yVan Ty, 1941), L. youngi, L. spinicrassa, L. evansi, L. ovallesi, L. longiflocosa, entre otras (34). Los haplotipos de ND4 han permitido diferenciar claramente hembras morfológicamente similares de $L$. youngiy $L$. spinicrassa, a través de porciones del gen que podrían considerarse, en algunos casos, como especie-específicas y potencialmente útiles para el diseño de sondas que permitan de una forma rápida y sencilla distinguir especies del complejo townsendi. El estudio ha sido complementado con ensayos de RFLP mediante la aplicación de algunas enzimas de restricción como VsP1 a los fragmentos amplificados del gen ND4. Los análisis de RFLPs se están realizando para verificar la existencia de bandas moleculares de tamaños específicos para algunas especies, que permitan no sólo discriminarlas sino también hacer reconstrucciones filogenéticas a partir del polimorfismo observado en los productos de PCR digeridos (55).

El gen citocromo b aparece como un marcador molecular útil para estudios de filogenética en Lutzomyia y Phlebotomus. Cáceres et al. 1999 (56) reportaron el uso secuencias de este gen y SRU para discriminar especies del subgénero Helcocyrtomyia Barretto, 1962: L. noguchii (Shannon, 1929); L. peruensis (Shannon, 1929); y L. tejadai Galati y Cáceres, 1990; y del subgénero Pifanomyia Ortiz y Scorza, 1963: L. verrucarum (Townsend, 1913); L. robusta Galati, Caceres y Le Pont, 1995; L. serrana (Damasceno y Arouck, 1949); y L. maranonensis Galati, Caceres y Le Pont, 1995. Como ha sido característico para genes ribosomales mitocondriales, las secuencias del gen SRU mostraron un menor porcentaje de divergencia nucleotídica que las secuencias de citocromo b, con una similaridad del $89 \%$ entre subgéneros y del 93 al $95 \%$ dentro de cada uno de estos. El gen citocromo b, por el contrario, presentó una similaridad del $79 \%$ entre subgéneros y del 82 al $86 \%$ dentro de cada subgénero, lo que les permitió concluir que citocromo b era, en este caso, el mejor marcador para distinguir las especies estrechamente relacionadas en ambos subgéneros. Secuencias nucleotídicas de este mismo gen están siendo usadas por Bejarano et al. 2000 (34), para estudios de filogenética entre varias especies del grupo verrucarum: $L$. ovallesi, L. youngi, L. spinicrassa, L. longiflocosa, $L$. columbiana, L. evansi y L. nuñeztovari (Ortiz, 1954).

En el género Phlebotomus, haplotipos del extremo 3 ' del gen citocromo b hasta la subunidad 1 de la $\mathrm{NADH}$ deshidrogenasa, separados por un gen ARN de transferencia (serina) y dos espaciadores intergénicos, fueron usados para estudiar las relaciones evolutivas en especies de los subgéneros Phlebotomus Rondani y Berté, 1840 y Larroussius Nitzulescu, 1931: P. papatasi; $P$. bergerotiParrot, 1934; $P$. duboscqi Neveu-Lemaire, 1906; P. perniciosus Newstead, 1911; P. tobbi Adler y Theodor, 1930; $P$. perfiliewi Parrot, 1930; y $P$. ariasi Tonnoir, 1921 (36). El alineamiento de las secuencias nucleotídicas produjo 20 haplotipos, que representaron a 21 poblaciones geográficas. Así mismo, el fragmento amplificado de 448 pb, reveló 124 sitios variables de los cuales 108 fueron filogenéticamente informativos, mostrando una tasa de divergencia nucleotídica uniforme entre las especies de ambos subgéneros. Esta fue estimada entre 1,0 y $2,5 \%$ por millón de años, para lo cual se calibró el reloj molecular mitocondrial tomando como referencia los eventos paleogeográficos ocurridos en África y la subregión mediterránea. Es importante resaltar que la filogenia inferida de los haplotipos mitocondriales en el subgénero Larroussius fue congruente con las relaciones previamente derivadas de caracteres morfológicos, y la divergencia en la secuencia nucleotídica fue compatible con eventos de especiación alopátrica originados por el aislamiento de poblaciones flebotomíneas del este y oeste del Viejo Mundo durante el Plioceno. 


\section{Conclusiones}

La utilización de genes mitocondriales para estudios taxonómicos en insectos de importancia médica ha tenido un auge inusitado durante los últimos años. Las primeras referencias bibliográficas sobre el uso de dichos genes en flebotomíneos, datan de 1997, fecha a partir de la cual se observa un crecimiento moderado en el número de publicaciones.

Aunque también se han empleado genes nucleares (subunidades ribosomales $18 S$ y $28 \mathrm{~S}$, espaciador interno transcrito 2) en la taxonomía de los géneros Phlebotomus y Sergentomyia (57$61)$, el uso del ADN mitocondrial se ha visto favorecido, entre otras razones, por la utilidad de algunas regiones tanto para análisis de variabilidad intraespecífica como interespecífica, y por la disponibilidad de oligonucleótidos conservados que facilitan la amplificación de éstas en un número casi ilimitado de especies. Esto explica la amplia acogida que han tenido los genes SRU, LRU, COI, ND4 y citocromo b. La selección de este pequeño número de marcadores mitocondriales ampliamente aceptados, permitirá realizar en un futuro, macrorreconstrucciones de la crónica evolutiva de los flebotomíneos, con base en las secuencias nucleotídicas acumuladas por los distintos autores en bases de datos como Genbank/National Center for Biotechnology Information (NCBI), European Molecular Biology Laboratory (EMBL) y DNA Data Bank of Japan (DDBJ).

Además de genes mitocondriales, es necesario considerar la inclusión de algunos genes nucleares en el estudio de las especies flebotomíneas americanas, con el objetivo de validar y otorgar mayor robustez a las filogenias derivadas del ADN mitocondrial. Los marcadores nucleares por utilizar deberán ser cuidadosamente seleccionados, ya que la existencia de múltiples copias de algunas regiones nucleares como las ribosomales plantea la posibilidad de la presencia de heteroplasmia, es decir, polimorfismo dentro de un mismo individuo, el cual afecta la información que pueda derivarse de ellos.

La literatura revisada muestra que la principal aplicación de los genes mitocondriales en vectores de leishmaniosis, ha estado en estudios de variación intraespecífica o genética de poblaciones. Esto puede explicarse por el interés que han despertado recientemente complejos de especies como townsendi, $L$. longipalpis y $L$. whitmani. Sin embargo, las reconstrucciones filogenéticas y evolutivas en flebotomíneos con base en secuencias de ADN mitocondrial, aunque se encuentran en una etapa inicial, serán probablemente las que más aportarán a la identificación y clasificación de estos insectos.

Los genes mitocondriales aun cuando no son el único medio para diferenciar especies morfológicamente similares y realizar reconstrucciones filogenéticas, sí se muestran en la actualidad como una herramienta adecuada que permite obviar los problemas morfológicos y quizás, en un futuro, con la ayuda de otros marcadores moleculares contribuirá en la resolución de las disputas taxonómicas que han caracterizado a los miembros de la subfamilia Phlebotominae durante años.

\section{Agradecimientos}

El autor expresa sus agradecimientos a Sandra Uribe por su asesoría en el estudio molecular del grupo verrucarum y al entomólogo Winston Rojas por sus aportes para el mejoramiento del manuscrito.

El trabajo con verrucarum fue financiado por el Instituto Colombiano para el Desarrollo de la Ciencia y la Tecnología Francisco José de CaldasCOLCIENCIAS (proyecto 1115-05-106-97), y el Comité para el Desarrollo de la InvestigaciónCODI, Universidad de Antioquia (proyecto СРT0019).

\section{Referencias}

1. Williams P. Relationships of phlebotomine sand flies (Diptera). Mem Inst Oswaldo Cruz 1993;88:177-83.

2. Lewis DJ, Young DG, Fairchild GB, Minter DM. Proposal for a stable classification of the phlebotomine sandflies (Diptera: Psychodidae). Syst Entomol 1977;2: 319-32.

3. Lanzaro GC, Warburg A. Genetic variability in phlebotomine sandflies: possible implications for leishmaniasis epidemiology. Parasitol Today 1995;11: 151-4.

4. Theodor $\mathbf{O}$. On the classification of American Phlebotominae. J Med Entomol 1965;2:171-97. 
5. Young DG, Duncan MA. Guide to identification and geographic distribution of Lutzomyia sand flies in Mexico, the West Indies, Central and South America (Diptera: Psychodidae). Mem Amer Entomol Inst 1994;54:1-881.

6. Kreutzer RD, Palau MT, Morales A, Ferro C, Feliciangeli D, Young DG. Genetics relationships among phlebotomine sand flies (Diptera: Psychodidae) in the verrucarum species group. J Med Entomol 1990; $27: 1-8$.

7. Anez N, Valenta DT, Cazorla D, Quicke DJ, Feliciangeli MD. Multivariate analysis to discriminate species of phlebotomine sand flies (Diptera: Psychodidae): Lutzomyia townsendi, L. spinicrassa, and L. youngi. J Med Entomol 1997;34:312-6.

8. Young DG, Morales A, Kreutzer RD, Alexander JB, Corredor A, Tesh RB, et al. Isolations of Leishmania braziliensis (Kinetoplastida: Trypanosomatidae) from cryopreserved Colombian sand flies (Diptera: Psychodidae). J Med Entomol 1987;24:587-9.

9. Feliciangeli D, Arredondo C, Ward R. Phlebotomine sandflies in Venezuela: review of the verrucarum species group (in part) of Lutzomyia (Diptera: Psychodidae) with description of a new species from Lara. J Med Entomol 1992;29:729-44.

10. Santamaría E, Castillo M, Cárdenas R, Bello F, Ayala M, Ferro C. Competencia vectorial de las especies de Lutzomyia del grupo verrucarum (Diptera: Psychodidae) en un foco endémico de Leishmania braziliensis en Reventones, Cundinamarca. Biomédica 1999;19:115-26.

11. Artemiev MM. A classification of the subfamily Phlebotominae. Parassitologia 1991;33(Suppl.1):69-77.

12. Martins AV, Williams P, Falcão AL. American sand flies (Diptera: Psychodidae, Phlebotominae). Rio de Janeiro, Brasil: Academia Brasiliera de Ciências; 1978. p.1-195.

13. Galati BEA. Phylogenetic systematics of Phlebotominae (Diptera, Psychodidae) with emphasis on American groups. Bol Dir Malariol y San Amb 1995;35(Suppl.1):13342.

14. Dujardin JP, Le Pont F, Martinez E. Quantitative phenetics and taxonomy of some phlebotomine taxa. Mem Inst Oswaldo Cruz 1999;94:735-41.

15. Saiki RK, Scharf S, Faloona F, Mullis KB, Horn GT, Erlich HA, et al. Enzymatic amplification of B-globin genomic sequences and restriction site analysis for diagnosis of sickle cell anemia. Science 1985;230:1350-4.

16. Smith LM, Sanders JZ, Kaiser RJ, Hughes P, Dodd C, Connell CR, et al. Fluorescence detection in automated DNA sequence analysis. Nature1986;321:674-9.

17. Paskewitz SM, Collins FH. Use of the polymerase chain reaction to identify mosquito species of the Anopheles gambiae complex. Med Vet Entomol 1990;4:367-73.

18. García BA, Powell JR. Phylogeny of species of Triatoma (Hemiptera: Reduviidae) based on mitochondrial DNA sequences. J Med Entomol 1998;35:232-8.
19. Moulton JK. Molecular sequence data resolves basal divergences within Simulidae (Diptera). Syst Entomol 2000;25:95-113.

20. Avise JC. Molecular markers, natural history and evolution. $1^{\text {st }}$ ed. New York: Chapman \& Hall, Inc.; 1994. p.1-511.

21. Tegelström $\mathbf{H}$. Detection of mitochondrial DNA fragments. En: Hoelzel AR, editor. Molecular genetic analysis of populations. A practical approach. Oxford, New York, Tokyo: Oxford University Press; 1992. p.29-58.

22. Beard CB, Hamm DM, Collins FH. The mitochondrial genome of the mosquito Anopheles gambiae: DNA sequence, genome organization, and comparisons with mitochondrial sequences of other insects. Insect Mol Biol 1993;2:103-24.

23. Lodish H, Baltimore D, Berk A, Lawrence S, Matsudaira P, Darnell J. Molecular cell biology. $3^{\text {rd }}$ ed. New York: Scientific American Books, Inc; 1995. p.11344.

24. Goddard JM, Wolstenholme DR. Origin and direction of replication in mitochondrial DNA molecules from Drosophila melanogaster. Proc Natl Acad Sci USA 1978;76: 3886-90.

25. Lessinger AC, Azeredo-Espin ML. Evolution and structural organisation of mitochondrial DNA control region of myiasis-causing flies. Med Vet Entomol 2000;14: 71-80.

26. Uribe S, Porter C, Vélez ID. Amplificación y obtención de secuencias de rRNA mitocondrial en Lutzomyia spp. (Diptera: Psychodidae) vectores de leishmaniosis. Socolen 1998;24:109-15.

27. Crozier RH, Crozier YC. The mitochondrial genome of the honeybee Apis mellifera: complete sequence and genome organization. Genetics 1993;133:97-117.

28. Moritz C, Hillis DM. Molecular systematics context and controversies. En: Hillis DM, Moritz C, Mable BK, editors. Molecular Systematics. $2^{\text {nd }}$ ed. Sunderland, Massachusetts: Sinauer Associates, Inc.; 1996. p.1-13.

29. Caterino MS, Cho S, Sperling FA. The current state of insect molecular systematics: a thriving Tower of Babel. Annu Rev Entomol 2000;45:1-54.

30. Harrison R. Animal mitochondrial DNA as a genetic marker in population and evolutionary biology. Tree 1994;4:6-11.

31. Simon C, Frati F, Beckenbach A, Crespi B, Liu H, Flook P. Evolution, weighting, and phylogenetic utility of mitochondrial gene sequences and a compilation of conserved polymerase chain reaction primers. Ann Entomol Soc Am 1994;87:651-701.

32. Mitchell SE, Cockburn AF, Seawright JA. The mitochondrial genome of Anopheles quadrimaculatus species A: complete nucleotide sequence and gene organization. Genome 1993;36:1058-73. 
33. Ready PD, Day JC, De Souza AA, Rangel EF, Davies CR. Mitochondrial DNA characterization of populations of Lutzomyia whitmani (Diptera: Psychodidae) incriminated in the peri-domestic and silvatic transmission of Leishmania species in Brazil. Bull Entom Res 1997;87: 187-95.

34. Bejarano E, Rojas W, Uribe S, Porter C, Vélez ID. Phylogenetics and evolution of Lutzomyia spp. (verrucarum group) in the Andean regions of Colombia. Abstracts Vol. 2, XV International Congress for Tropical Medicine and Malaria, Cartagena; 2000. p.130.

35. Uribe S, Lehmann T, Rowton ED, Vélez ID, Porter C. Speciation and population structure in the morphospecies Lutzomyia longipalpis (Lutz \& Neiva) as derived from the mitochondrial ND4 gene. Mol Phylogenet Evol 2001;18:456-61.

36. Esseghir S, Ready PD, Killick-Kendrick R, BenIsmail R. Mitochondrial haplotypes and phylogeography of Phlebotomus vectors of Leishmania major. Insect Mol Biol 1997;6:211-25.

37. Sanjoba C, Özbel Y, Yamauchi M, Sasaki T, Ito M, Matsumoto $\mathrm{Y}$, et al. Nucleotide sequence variations of the cytochrome c oxidase subunit I gene within Phlebotomus species, vector insects for leishmaniasis. Abstracts, $3^{\text {rd }}$ International Symposium of Phlebotomine Sandflies, Montpellier, France; 1999. p.033.

38. HsuChen CC, Kotin RM, Dubin DT. Sequences of the coding and flanking regions of the large ribosomal subunit RNA gene of mosquito mitochondria. Nucleic Acids Res 1984;12:7771-85.

39. Gorrochotegui-Escalante N, Muñoz ML, Fernández-Salas I, Beaty BJ, Black WC. Genetic isolation by distance among Aedes aegyptipopulations along the northeastern coast of Mexico. Am J Trop Med Hyg 2000;62:200-9.

40. Ho CM, Liu YM, Wei YH, Hu ST. Gene for cytochrome c oxidase subunit II in the mitochondrial DNA of Culex quinquefasciatus and Aedes aegypti (Diptera: Culicidae). J Med Entomol 1995;32:174-80.

41. Guillemaud T, Pasteur N, Rousset F. Contrasting levels of variability between cytoplasmic genomes and incompatibility types in the mosquito Culex pipiens. Proc $\mathrm{R}$ Soc Lond B Biol Sci 1997;264:245-51.

42. Xiong B, Kocher TD. Phylogeny of sibling species of Simulium venustum and S. verecundum (Diptera: Simuliidae) based on sequences of the mitochondrial 16 SrRNA gene. Mol Phylogenet Evol 1993;2:293-303.

43. Tang J, Toe L, Back C, Zimmerman PA, Pruess K, Unnasch TR. The Simulium damnosum species complex: phylogenetic analysis and molecular identification based upon mitochondrially encoded gene sequences. Insect Mol Biol 1995;4:79-88.

44. Monteiro FA, Perez R, Panzera F, Dujardin JP, Galvao C, Rocha D, et al. Mitochondrial DNA variation of
Triatoma infestans populations and its implication on the specific status of T. melanosoma. Mem Inst Oswaldo Cruz 1999;94(Suppl.1):229-38.

45. Lyman DF, Monteiro FA, Escalante AA, CordonRosales C, Wesson DM, Dujardin JP, et al. Mitochondrial DNA sequence variation among triatomine vectors of Chagas' disease. Am J Trop Med Hyg 1999;60:37786.

46. Quicke DLJ. Principles and techniques of contemporary taxonomy. $1^{\text {st }}$ ed. London: Blackie Academic \& Professional; 1993. p.1-311.

47. Uribe S. Polimorfismo entre poblaciones de Lutzomyia longipalpis, insecto transmisor de leishmaniosis visceral, sugiere la existencia de un complejo de especies. Biomédica 1999;19:56-66.

48. Arrivillaga JC, Norris DE, Lanzaro GC. Intraspecific phylogenetic analysis of Lutzomyia longipalpis (Diptera: Psychodidae) using mitochondrial DNA. Abstracts, $3^{\text {rd }}$ International Symposium of Phlebotomine Sandflies, Montpellier, France; 1999. p.W6.

49. Bejarano EE. Estructura poblacional y variabilidad genética en vectores de leishmaniosis: Lutzomyia spp. (grupo verrucarum). latreia 2000;13:109.

50. Montoya-Lerma J, Ready PD, Testa JM, Oviedo M, Lane RP. Molecular systematics of Lutzomyia evansi and the verrucarum group. Abstracts, $3^{\text {rd }}$ International Symposium of Phlebotomine Sandflies, Montpellier, France; 1999. p.P47.

51. Ready PD, De Souza AA, Rebelo JM, Day JC, Silveira FT, Campbell-Lendrum D, et al. Phylogenetic species and domesticity of Lutzomyia whitmani at the southeast boundary of Amazonian Brazil. Trans R Soc Trop Med Hyg 1998;92:159-60.

52. Ishikawa EA, Ready PD, De Souza AA, Day JC, Rangel EF, Davies CR, et al. A mitochondrial DNA phylogeny indicates close relationships between populations of Lutzomyia whitmani (Diptera: Psychodidae, Phlebotominae) from the rain-forest regions of Amazonia and northeast Brazil. Mem Inst Oswaldo Cruz 1999;94: 339-45.

53. Futuyma DJ. Evolutionary biology. $3^{\text {rd }}$ ed. Sunderland, Massachusetts: Sinauer Associates, Inc; 1998. p.1-763.

54. Suguri S, Harada M, Maruno M, Hosokawa A, Sud R, Gómez EA, et al. A preliminary study on nucleotide sequence variations of Lutzomyia spp. in the cytochrome c oxidase subunit I gene. En: Hashiguchi Y, editor. Studies on New World leishmaniasis and its transmission, with particular reference to Ecuador. Kochi, Japan: Kyowa Printing \& Co. Ltd; 1997. p.25-7.

55. Rojas W, Uribe S, Bejarano E, Porter C, Vélez ID. Molecular search of markers for the diagnosis of species of Lutzomyia spp. of the verrucarum group (Theodor, 1965). Abstracts Vol. 2, XV International Congress for Tropical Medicine and Malaria, Cartagena; 2000. p.131. 
56. Cáceres AL, Regnery RL, Beati L. Molecular tools for the taxonomic and phylogenetic study of Lutzomyia spp. Abstracts, $3^{\text {rd }}$ International Symposium of Phlebotomine Sandflies, Montpellier, France; 1999. p.P48.

57. Depaquit J, Ferte H, Leger N. The subgenus Paraphlebotomus (Phlebotomus - Phlebotominae - Psychodidae - Diptera): a review. Morphological and molecular studies. Ann Pharm Fr 2000;58:333-40.

58. Depaquit J, Ferte H, Leger N, Killick-Kendrick R, Rioux JA, Killick-Kendrick $M$, et al. Molecular systematics of the phlebotomine sandflies of the subgenus Paraphlebotomus (Diptera, Psychodidae, Phlebotomus) based on ITS2 rDNA sequences. Hypotheses of dispersion and speciation. Insect Mol Biol 2000;3:293-300.
59. Aransay AM, Scoulica E, Tselentis Y, Ready PD. Phylogenetic relationships of phlebotomine sandflies inferred from small subunit nuclear ribosomal DNA. Insect Mol Biol 2000;9:157-68.

60. Aransay AM, Scoulica E, Chaniotis B, Tselentis Y. Typing of sandflies from Greece and Cyprus by DNA polymorphism of $18 \mathrm{~S}$ rRNA gene. Insect Mol Biol 1999;8: 179-84.

61. Di Muccio T, Marinucci M, Frusteri L, Maroli M, Pesson B, Gramiccia M. Phylogenetic analysis of Phlebotomus species belonging to the subgenus Larroussius (Diptera, Psychodidae) by ITS2 rDNA sequences. Insect Biochem Mol Biol 2000;30:387-93. 